\title{
Anatomía y Competencias Clínicas en Odontología. Estudio Basado en Apreciación de Estudiantes
}

\author{
Anatomy and Clinical Competences in Dentistry. \\ Study Based on Students Appreciation \\ *Fernando García-Hernández \& **íctor Aravena Verdejo
}

GARCÍA-HERNÁNDEZ, F. \& ARAVENA, V. V. Anatomía y competencias clínicas en Odontología. Estudio basado en apreciación de estudiantes. Int. J. Morphol., 24(3):443-448, 2006.

RESUMEN: La Anatomía entrega a los estudiantes conceptos sobre la estructura, pero sobre todo un método de estudio y una nomenclatura que serán usados en asignaturas básicas y clínicas, motivo por lo que la disciplina se enseña en los primeros años de la Carrera. La educación basada en competencias enfatiza el producto final y define lo que debe saber el alumno al finalizar su formación, independiente de cómo lo enseñó el profesor o los sistemas de aprendizaje que utilizó. Las competencias finales determinan lo que se ha de enseñar e identifican lo esencial de lo superfluo. Representa el mejor método para acreditar la calidad del "producto elaborado" por cada institución de Educación Superior, el profesional que se envía al medio para cumplir su rol en la comunidad. Las competencias y habilidades del Cirujano Dentista general, dependerán de las exigencias que se establezcan en el ámbito público y privado para su desempeño profesional. La presente investigación pretende obtener información de todos los estudiantes que cursan las asignaturas clínicas de Odontología en la Universidad de Antofagasta (n=95), referente a apreciación del nivel curricular de la asignatura, la continuidad del aprendizaje, medios de reforzamiento y comprobar el factor de olvido de la Anatomía, mediante un cuestionario de preguntas básicas para realizar competencias clínicas. Los resultados de la evaluación demostraron que ningún curso alcanzó el $60 \%$ de rendimiento mínimo exigido. La mayoría de los alumnos recomiendan que la asignatura de Anatomía General se mantenga en el primer nivel curricular y que se establezca una asignatura de Anatomía de Cabeza y Cuello en cursos superiores, además, que en cada curso clínico, un anatomista aporte el enfoque anatómico requerido. Se destacan los medios de reforzamiento utilizados y la apreciación personal del conocimiento anatómico que se disponía al momento de iniciar cursos clínicos.

PALABRAS CLAVES: Anatomía; Odontología; Educación.

\section{INTRODUCCIÓN}

En forma tradicional, la asignatura de Anatomía se dicta generalmente en los dos primeros niveles de la Carrera de Odontología, situación que dificulta su integración con las asignaturas clínicas, no permitiendo que en niveles de inicio, los estudiantes comprendan la relevancia que la disciplina posee para desarrollar las competencias clínicas y profesionales que deberán cumplir durante su entrenamiento de pregrado (Comité de Expertos de la OMS/OPS en la Enseñanza de la Morfología, 1970; DiDio, 1973; Sociedad Anatómica Española, 1996).

En la carrera de Odontología de la Universidad de Antofagasta, la asignatura de Anatomía se desarrolla en forma anual durante el primer nivel curricular, con ocho horas de carga académica y cuyos contenidos están divididos en tres unidades temáticas: Anatomía Sistémica, Anatomía de
Cabeza y Cuello y Anatomía Bucodentaria, durante las cuales se aportan conceptos de aplicación clínica y quirúrgica.

A contar del año 2004, la Universidad de Antofagasta ha decidido aplicar un modelo pedagógico centrado en el alumno y cuyos objetivos terminales se basan en el logro de competencias, y que, en lo referente a Ciencias Médicas, esta teoría educacional fue desarrollada por Harden y colaboradores en la Universidad de Dundee en Escocia, Reino Unido (Universidad de Chile Facultad de Medicina, Departamento de Educación en Ciencias de la Salud, Escuela de Postgrado, 2003; Universidad de Antofagasta CIAE, 2004).

Se puede definir competencias como aquellas habilidades que tiene que dominar todo profesional y que emanan del ejercicio de su profesión.

\footnotetext{
* Unidad de Anatomía Departamento Biomédico, Facultad de Ciencias de la Salud, Universidad de Antofagasta, Chile.

**Departamento de Odontología, Facultad de Medicina y Odontología, Universidad de Antofagasta, Chile.
} 
La definición de Kane (1992) en Brailovsky, (2002) parece representar una de las definiciones más dinámicas e interesantes. Este autor define competencia profesional como: "... el grado de utilización de los conocimientos, las habilidades y el buen juicio asociados a la profesión, en todas las situaciones que se pueden confrontar en el ejercicio de la práctica profesional".

Las competencias y habilidades que debe poseer un Cirujano Dentista General, dependerán de las exigencias que se le exija en su desempeño profesional en los ámbitos público y privado.

Cada institución de educación superior debe definir el perfil profesional que desea que sus egresados posean al salir al medio laboral, y, que de alguna manera, representa la impronta formativa lograda al finalizar sus estudios de pregrado.

La Universidad de Antofagasta ha establecido las competencias generales que deben lograr sus estudiantes, a su vez, cada carrera de acuerdo al perfil profesional de egreso determinó las competencias profesionales específicas y donde cada asignatura aporta las habilidades y conocimientos necesarios para el logro de dichas competencias.

La Unión Europea (UE) a través del proyecto DentEd (European Dental Education), la Federación Dental Internacional (FDI) y, en Chile, la Comisión Nacional de Acreditación del Pregrado (CNAP) y la Asociación Chilena de Escuelas de Odontología (ACHEO) han elaborado listados de competencias que debe poseer cada graduado en Odontología al egresar, cada una de las cuales requiere de conocimientos básicos, clínicos, técnicos y de formación general.

El aporte de la Anatomía según la educación basada en las competencias, de acuerdo al mismo informe del proyecto DentEd, señala que "debe ser la adecuada para proveer un detallado conocimiento de la Anatomía relevante para la práctica como Cirujano Dentista combinada con una visión general de la anatomía humana necesaria para la comprensión de la fisiología y de la patología del cuerpo humano".

El propósito de la presente investigación es obtener información de parte de los estudiantes que cursan las asignaturas clínicas de Odontología en la Universidad de Antofagasta, referente a su apreciación respecto a:

1) Cuándo debe impartirse el conocimiento anatómico que sirva para desarrollar las competencias clínicas.

2) El nivel de profundidad con que debe dictarse los conceptos de aplicación clínica durante el primer año de la carrera.
3) La importancia de la continuidad del aprendizaje para evitar el factor de olvido.

4) La necesidad de integración con las asignaturas propiamente profesionales.

\section{MATERIAL Y MÉTODO}

El grupo de estudio corresponde a todos los alumnos que cursan cuarto, quinto y sexto años de la Carrera. A todos los estudiantes se les aplicó una prueba de diagnóstico anatómico con 10 preguntas, cinco de respuesta breve y cinco de selección múltiple además de un cuestionario autoadministrado, confiable y validado, todo lo cual servirá para verificar el conocimiento anatómico y explorar las causas que los alumnos consideran determinantes en la retención del conocimiento anatómico al iniciar las actividades clínicas. Una vez revisada y calificada la evaluación y tabulados los datos del cuestionario se realizará análisis estadístico mediante programas Microsoft Excel 7.0 y Statgraphics 4.0.

\section{RESULTADOS Y DISCUSIÓN}

El análisis de los resultados se dividió en dos partes. En la primera de ellas se analizaron los resultados obtenidos de la evaluación aplicada a los estudiantes y en la segunda parte se relacionaron entre sí estos últimos y los antecedentes aportados en la encuesta anexa.

\section{Análisis de los resultados obtenidos en evaluación ana-} tómica. Durante los estudios regulares de la Carrera de Odontología, el estudiante debe cumplir a lo menos el $60 \%$ de los objetivos establecidos por la asignatura, para lograr la aprobación de la asignatura de Anatomía. El resultado de la presente evaluación diagnóstica aplicada a los estudiantes de cursos clínicos de la Carrera, demostró que el promedio del rendimiento de cada curso fue inferior a este porcentaje $(\mathrm{n}=$ 34 en $4^{\circ}$ año, promedio $50 \% ; n=42$ en $5^{\circ}$ año, promedio $51,1 \%$; $n=19$ en $6^{\circ}$ año, promedio $48,9 \%$ ), no comprobándose diferencias estadísticamente significativas (95\%) entre los 3 niveles (Fig. 1). El promedio porcentual obtenido de todos los alumnos fue de 50,3\% ( $n=95)$, no alcanzándose en ningún curso el porcentaje mínimo de aprobación.

Relación entre resultados de evaluación anatómica y opinión de estudiantes. El 61,1\% de los estudiantes (Fig. 2) considera que en la asignatura de Anatomía se entregaron los conocimientos necesarios para desarrollar los diversos procedimientos exigidos en las asignaturas clínicas $(17,9 \%$ 


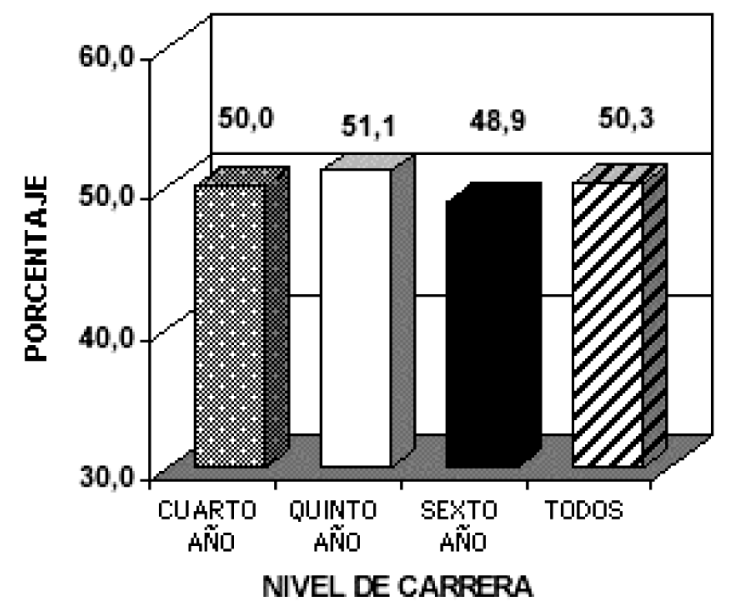

Fig. 1. Porcentaje promedio de respuestas correctas Test de Anatomía aplicado a alumnos de asignaturas clínicas, $\mathrm{Ca}$ rrera de Odontología de la Universidad de Antofagasta, Chile. $(n=95)$.

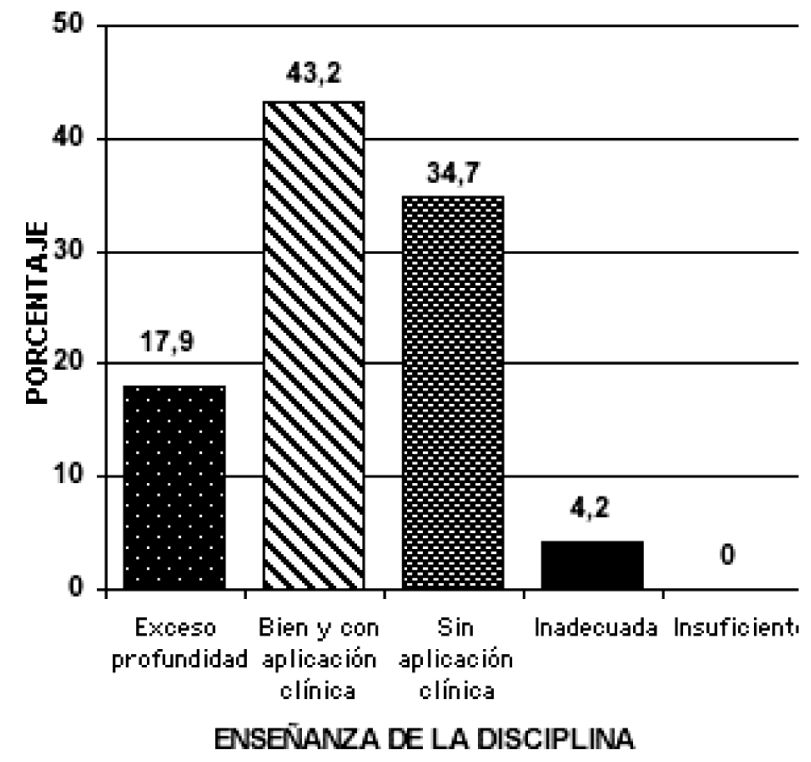

Fig. 2. Porcentaje de opinión de alumnos de Odontología de la Universidad de Antofagasta, Chile, respecto de la docencia de Anatomía entregada en Primer Año y su posterior aplicación en cursos clínicos $(n=95)$.

con excesiva profundidad y $43,2 \%$ bien y con aplicación clínica). El grupo de estudiantes que indicaron que la Anatomía fue bien enseñada y con aplicación clínica, obtuvieron el porcentaje más alto de respuestas correctas en la evaluación anatómica (Fig. 3), estando cercano al porcentaje de aprobación mínimo $(54,6 \%$ de $60 \%)$, siendo la diferencia porcentual estadísticamente significativa al $90 \%$.

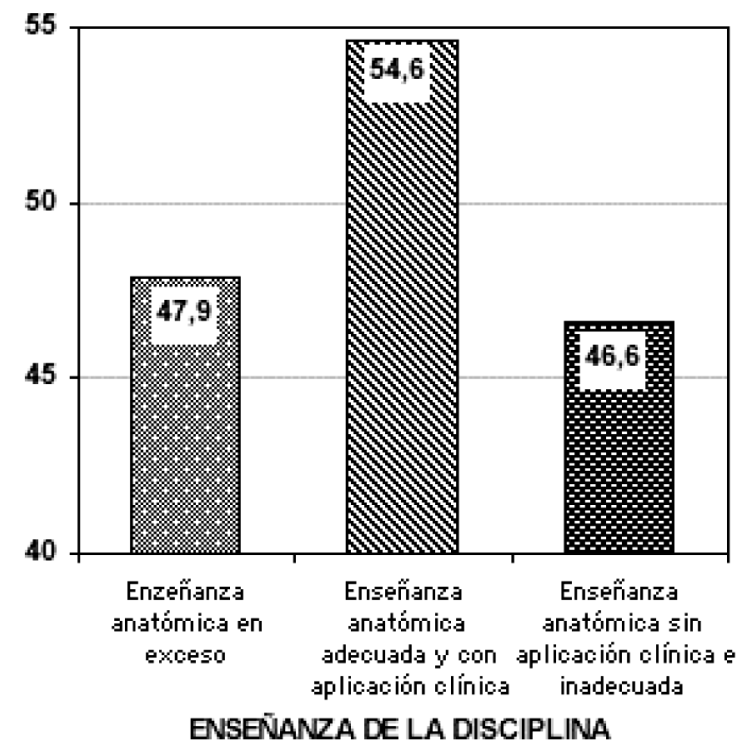

Fig. 3. Comparación de promedios Test de Anatomía y opinión del alumno sobre la enseñanza de la Anatomía, Carrera de Odontología, Universidad de Antofagasta, Chile $(\mathrm{n}=93)^{* * * * *} 2$ alumnos no contestaron la pregunta.

El 57,9\% de los estudiantes señala que, según su percepción, los conocimientos anatómicos que poseían eran insuficientes al iniciar las diversas asignaturas clínicas de la Carrera (Fig. 4).

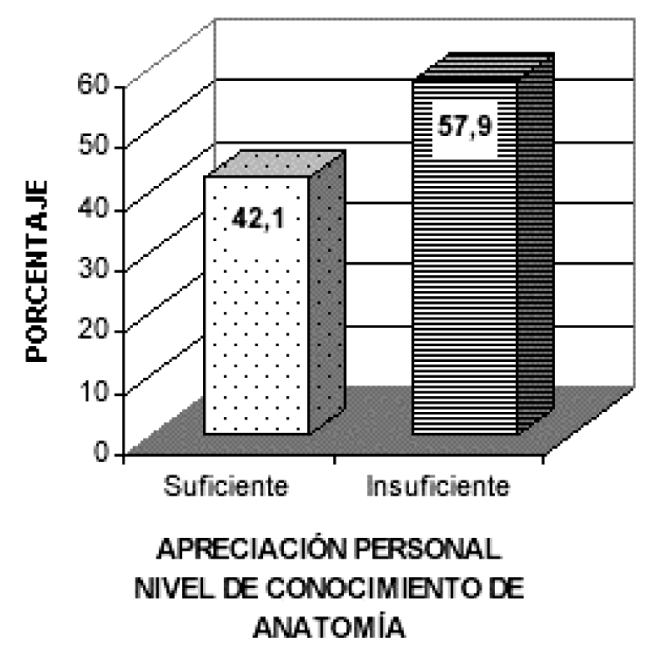

Fig. 4. Porcentaje de encuesta de opinión sobre suficiencia de conocimientos anatómicos que poseen los alumnos de Odontología de la Universidad de Antofagasta, Chile al iniciar asignaturas clínicas $(\mathrm{n}=95)$. 
En la Fig. 5 se comparan la apreciación personal de los estudiantes respecto de la enseñanza anatómica en primer año de acuerdo a los requerimientos clínicos, la percepción del nivel personal de conocimientos anatómicos al iniciar las diversas asignaturas clínicas y el promedio obtenido en el test de Anatomía aplicado. Se observa una correlación entre la apreciación personal de su cono- cimiento, el nivel de enseñanza anatómico y el resultado del test, con excepción del segundo grupo que pese a su suposición de poseer conocimientos anatómicos insuficientes y, considerando adecuada la enseñanza de la disciplina, obtienen un 54,2\% de respuestas correctas en el test, lo que demuestra una base anatómica mejor que su apreciación personal.

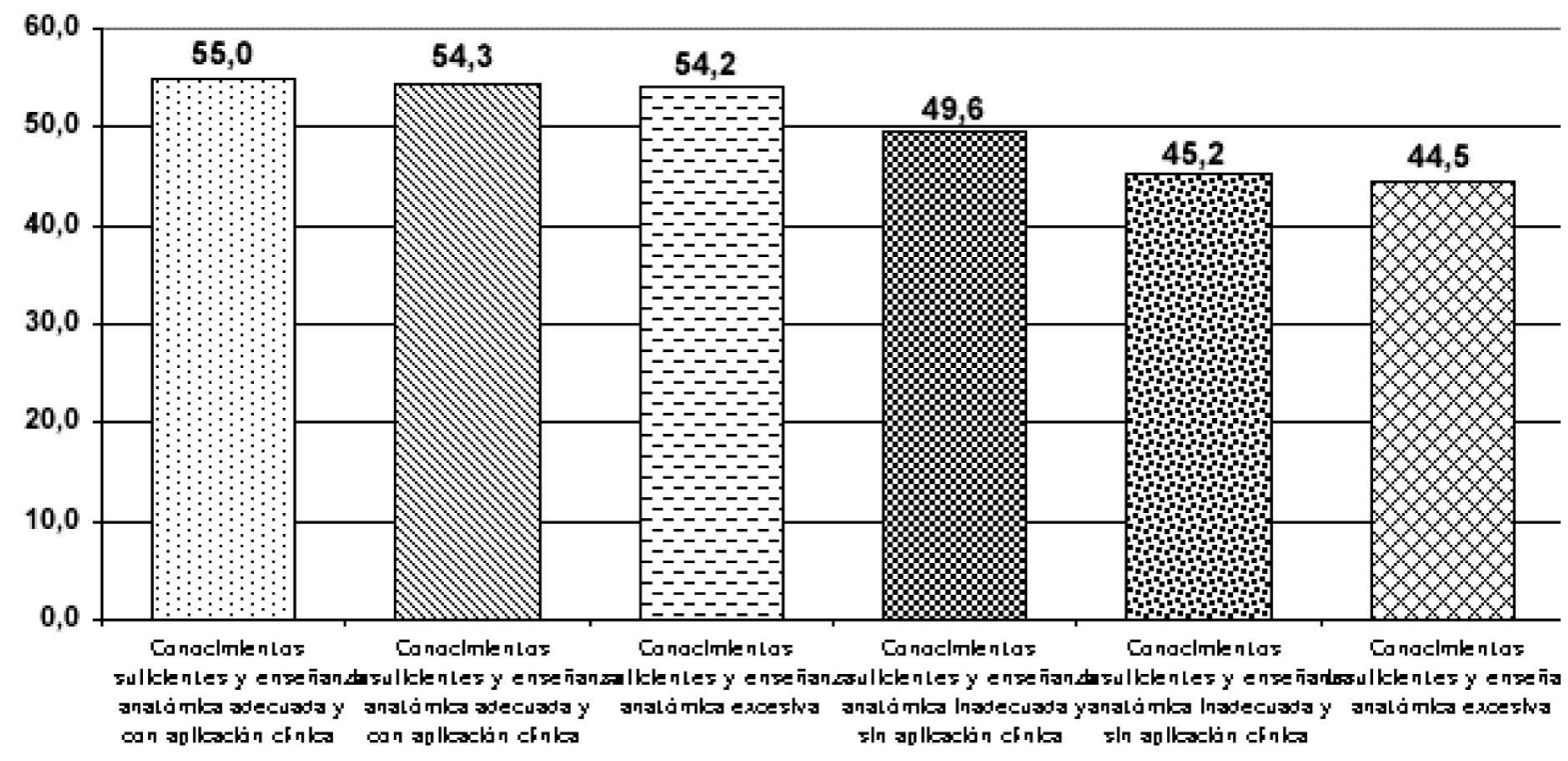

APRECIACIÓN PERSONAL DE CONOCIMIENTOS Y NIVEL DE ENSEÑANZA DE LA ANATOMIAA

Fig. 5. Comparación de promedios de respuestas correctas Test de Anatomía a percepción personal del tipo de enseñanza anatómica y de conocimientos anatómicos al iniciar clínicas. Alumnos de Odontología de la Universidad de Antofagasta, Chile. (n=95).

Como reforzamiento del conocimiento anatómico para iniciar actividades clínicas, los estudiantes señalan su preferencia por la revisión de textos o atlas de la disciplina, no existiendo diferencias estadísticamente significativas entre los diversos métodos de reforzamiento (Fig. 6).

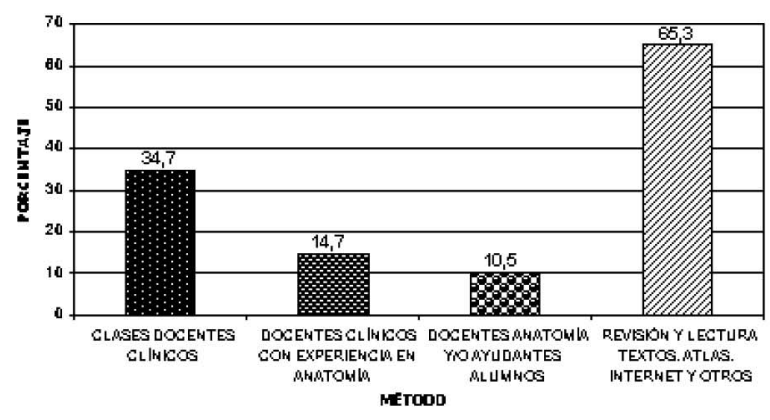

Fig. 6. Métodos de reforzamiento de Anatomía utilizados en asignaturas clínicas por alumnos de Odontología de la Universidad de Antofagasta ( $\mathrm{n}=95)$.
En la Fig. 7 se detalla la opinión de los estudiantes respecto de la ubicación curricular de la asignatura, donde la mayoría propone la dictación de una asignatura de Anatomía General en los primeros niveles de la Carrera y que, a su vez, se dicte una asignatura de Anatomía de Cabeza y

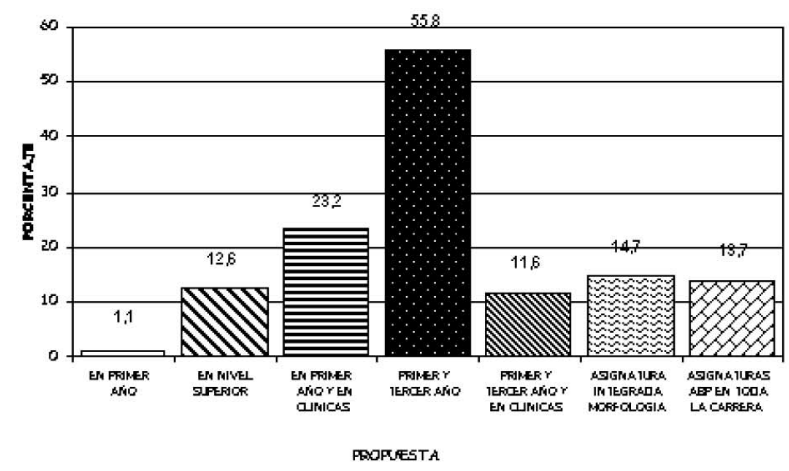

Fig. 7. Opinión de los alumnos que cursan asignaturas clínicas en la Carrera de Odontología de la Universidad de Antofagasta respecto del nivel de ubicación de las asignaturas de Anatomía General y Especializada $(n=95)$. 
Cuello en el tercer año de la Carrera $(55,8 \%)$ o en cursos superiores $(12,6 \%)$, no siendo excluyentes las otras propuestas que sugieren llevar hacia el nivel clínico la enseñanza específica de determinados conocimientos anatómicos.

Del análisis de los resultados obtenidos, se pueden extraer las siguientes conclusiones:

1) Se comprueba un marcado olvido de los conocimientos anatómicos entregados en el primer nivel de estudios por parte de los estudiantes de cursos clínicos, avalado y ratificado lo anterior, tanto por el resultado de la prueba de diagnóstico así como la percepción personal de los propios estudiantes. Estos resultados son similares a los obtenidos en una investigación similar, realizada en el año 1995, por uno de los autores del presente trabajo, en la Carrera de Medicina de la Universidad de Valparaíso (Maldonado et al., 1993).

2) Los estudiantes consideran mayoritariamente $(54,6 \%)$ que la asignatura de Anatomía dictada en el primer ni- vel de la Carrera, se enseñó adecuadamente y con aplicación clínica y donde estos estudiantes obtuvieron un mejor resultado en la prueba de diagnóstico, siendo estadísticamente significativa al $90 \%$ respecto de los otros grupos.

3) Pese a que la mayoría de los estudiantes considera que la asignatura de Anatomía que se enseñó en primer año era adecuada a las necesidades de la Carrera y con aplicación a la clínica, la distancia temporal entre la dictación de la asignatura y la real utilización de estos conocimientos, hacen recomendable una modificación del plan de estudios, ubicando la Anatomía General en el primer nivel y creando una asignatura de Anatomía de Cabeza y Cuello en niveles superiores de la Carrera $(55,8 \%)$.

4) Como método de reforzamiento de los conocimientos anatómicos, los alumnos señalan preferencia en primer lugar por la revisión bibliográfica de textos anatómicos y en segundo lugar clases dictadas por docentes clínicos.

GARCÍA-HERNÁNDEZ, F. \& ARAVENA, V. V. Anatomy and clinical competences in dentistry. Study based on students appreciation. Int. J. Morphol., 24(3):443-448, 2006.

SUMMARY: The Anatomy gives to the students concepts on the structure, but mainly a method of study and a nomenclature that will be used in clinical basic subjects. This is the main reason why the discipline is taught in the first years of the careers. The education based on competences emphasizes the product and defines what the student must know when finalizing, independent of how to the professor taught or the learning systems used. The final competences determine what it is had to teach and identify the essential of the superfluous things. It represents the best method to credit the quality of the "manufactured product" by each institution of higher education, the professional who is sent to fulfil his roll in the community. The competences and abilities of the surgeon general dentist will depend on the exigencies that settle down in the public and private sector for their professional performance. The present investigation tries to obtain data of all the students who attend the clinical subjects of dentistry in the University of Antofagasta ( $n=95$ ). It deals with the appreciation that they have respect to the curricular level of the subject, the continuity of the learning, means of reinforcing, and to verify the factor of forgetfulness of the anatomy by means of a questionnaire of basic questions to make clinical competences. The results of the evaluation demonstrated that no course reached the required minimum of $60 \%$. Most of the students recommend that the subject of General Anatomy stays in the first curricular level and that a subject of Anatomy of Head and Neck in superior courses settles down. In addition, that in each clinical course, an anatomist contributes the required anatomical approach. Stand out the means of reinforcing used and the personal appreciation of the anatomical knowledge that that each student has at the time of initiating clinical courses.

KEY WORDS: Gross Anatomy; Dentistry; Education.

\section{REFERENCIAS BIBLIOGRÁFICAS}

Brailovsky, C.A. Educación Médica, evaluación de las competencias. http://www.fmv.uba.org.ar/proaps/9pdf, 2002.

Comité de Expertos de la OMS/OPS en la Enseñanza de la Morfología. Enseñanza de la Morfología en las Escuelas de Medicina de la América Latina. Primer Informe. Serie Desarrollo de Recursos Humanos A. Educación Médica $N^{\circ}$ 9, Organización Panamericana de la Salud, Oficina Sanitaria Panamericana, Oficina Regional de la OMS, Washington , 1970.
European Dental Education. Core Knowledge. www.dented.org/parse.php3?file=content/ knowledge.html

DiDio,L. J. A. Systems of teaching anatomy and evaluation of students. Arch. Mex. Anat., 36 (23-43), 1971.

DiDio, L. J. A. Tendencias modernas en la educación médica y su repercusión en la enseñanza de la Anatomía. Educación médica y salud, 2:184-97, 1973. 
Federación Dental Internacional. Declaración de la FDI: Resultados (competencias) en el entrenamiento de estudiantes de pregrado. www: $\mid$ Exec OfficelStatements\} Approved by GAISpanish\Basic dental Training - SP Clinical Competences 2003 Version dated,18 September 2003.

Kane, M. T. The assessment of clinical competence. Evaluation and the health professions, 15:163-82, 1992.

Maldonado, L.; García, F.; Goya, T.; De la Rosa, A.; Rosas, J. \& Pérez. E.: Determinación del grado de aprendizaje anatómico durante los años de estudio de la Carrera de Medicina. Rev. Chil. Anat., 11(2):84, 1993.

Sociedad Anatómica Española. II Jornadas sobre Enseñanza de la Anatomía. Bases para los programas en los planes de estudio de las diferentes Licenciaturas y Diplomaturas. Patrocinado por Sociedad Anatómica Española. Universidad Complutense, Facultad de Medicina, Madrid 10 de Mayo de 1996.

Universidad de Antofagasta, Facultad de Ciencias de la Salud. Plan de Estudios Carrera de Odontología, 2000.

Universidad de Antofagasta, Facultad de Ciencias de la Salud, Departamento Biomédico, Unidad de Anatomía. Programa de asignatura BI119 Anatomía Humana para Carrera de Odontología, 2003.

Universidad de Antofagasta. CIAE Documentos para acreditación de carreras. Antofagasta, 2004.

Universitat de Barcelona. Facultad de Medicina. Competencias a adquirir por los estudiantes de Medicina durante el pregrado en la Facultad de Medicina de la Universitat deBarcelona. Www.ub.es/medicina/castella/ compcast.pdf

Universidad de Chile, Facultad de Medicina, Departamento de Educación en Ciencias de la Salud, Escuela de Postgrado. Diploma en Ciencias Biomédicas. Módulo Currículum: Principios, tendencias y estrategias para su desarrollo, 2003.
Dirección para correspondencia: Prof. Dr. Fernando García Hernández Unidad de Anatomía. Departamento Biomédico Facultad de Ciencias de la Salud Universidad de Antofagasta CHILE

Email:fgarcia@uantof.cl

Recibido : 29-05-2006

Aprobado: 17-07-2006 